\title{
Prostate histotripsy: evaluation of prostatic urethral treatment parameters in a canine model
}

\author{
George R. Schade*, Nicholas R. Styn*, Kimberly A. Ives ${ }^{\dagger}$, Timothy L. Hall ${ }^{\dagger}$ and \\ William W. Roberts*†
}

Departments of *Urology and 'Biomedical Engineering, University of Michigan, Ann Arbor, MI, USA

\section{Objective}

- To assess the impact of histotripsy treatment parameters (pulse number and pulse-repetition frequency $[\mathrm{PRF}]$ ) on the efficiency of histotripsy induced homogenisation of the prostatic urethra.

\section{Materials and Methods}

- In all, 34 transabdominal prostate histotripsy treatments were applied along a perpendicular plane traversing the prostatic urethra of 21 dogs.

- Prostate histotripsy was applied with (i) escalating pulse number with fixed PRF or (ii) at fixed pulse number with varying PRFs.

- The development of urethral homognisation $\leq 14$ days of histotripsy was evaluated endoscopically and confirmed histologically.

\section{Results}

- Within 14 days of histotripsy $50 \%, 83 \%, 83 \%$, and $100 \%$ of dogs receiving 12 500, 25 000, 50 000, and 100000 pulses/mm of treatment path (delivered at $500 \mathrm{~Hz}$ PRF), respectively developed prostatic urethral disintegration.

- Delivery of 100000 pulses/mm was required to achieve urethral disintegration in all dogs within $24 \mathrm{~h}$ of histotripsy treatment.

- Increasing histotripsy PRF from 50 to 500 to $2000 \mathrm{~Hz}$ while applying a constant dose of 25000 pulses $/ \mathrm{mm}$ treatment was associated with increased rate of urethral disintegration ( $50 \%$ vs $75 \%$ vs $100 \%$ at 14 days, respectively).

\section{Conclusions}

- Increasing the number of histotripsy pulses and/or increasing the PRF of histotripsy treatment applied to the urethra may improve the rate and efficiency of prostatic urethral disintegration in the canine model.

- This understanding will aid in the development of treatment strategies for prostate histotripsy for benign prostatic hyperplasia in human trials.

\section{Keywords}

benign prostatic hyperplasia (BPH), focused ultrasound, prostate

\section{Introduction}

Histotripsy is a focused ultrasound technology that delivers short $(<50 \mu \mathrm{s})$, intense pulses of acoustic energy at low duty cycle to induce microbubble formation within a defined geometric focal volume while minimising thermal effects [1-5]. The oscillation and collapse of these bubbles mechanically homogenises targeted tissues into a fine slurry of acellular debris $[1,6,7]$. Analysis of the homogenate has revealed that $>99.9 \%$ of particles are $<6 \mu \mathrm{m}$ [8]. Volumetric ablation of greater than the size of a single focal volume is accomplished by moving the bubble cloud throughout the targeted region. Histotripsy therapy can be guided and monitored in real-time with conventional ultrasound because the bubble cloud appears as a distinct hyperechoic (bright) focus and targeted tissue echogenicity decreases (becomes darker) corresponding to progressive tissue disintegration $[9,10]$

The main objective of this research is to develop a histotripsy therapy for BPH that replicates the debulking capability of TURP, yet is less invasive and hence more widely applicable to patients with significant co-morbidity and/or suitable for use outside the operating room. Towards this goal, histotripsy has been evaluated in an in vivo canine model, demonstrating the ability to homogenize prostate tissue [11]. In this model, transabdominal histotripsy of the prostate appears safe and effective [12], producing dose-dependent tissue debulking of targeted prostatic tissue [13] with minimal haematuria, even in anticoagulated dogs [14]. The strategy for effective histotripsy treatment of BPH is to produce a TURP-like defect by homogenising parenchymal tissue as well as the adjacent 
prostatic urethra to facilitate drainage of the resultant homogenate with voiding [12]. However, the prostatic urethra is more resilient to mechanical forces than the prostate parenchyma and requires a greater number of acoustic pulses to achieve tissue disintegration [13]. In vitro studies have also shown that substrate stiffness is inversely correlated with histotripsy susceptibility and lesion size [15] and that altering the pulse-repetition frequency (PRF) can affect the efficiency of cavitation-induced tissue homogenisation [16]. As a result, the purpose of the present study was to explore the efficiency of prostatic urethra homogenisation in an in vivo canine model when varying the number and rate (PRF) of applied histotripsy pulses to better optimise treatment parameters in anticipation of future clinical applications.

\section{Material and Methods}

After receiving approval from the University Committee for Animal Use and Care, 21 intact male mongrel dogs weighing $25.0-33.6 \mathrm{~kg}$ were obtained. Intramuscular penicillin $\mathrm{G}$ benzathine (40 $000 \mathrm{IU} / \mathrm{kg}$ ) was administered before the procedure and on postoperative days (POD) 3 and 7 for prophylaxis. Carprofen $(2.2 \mathrm{mg} / \mathrm{kg} /$ day $)$ was administered orally before and for $24 \mathrm{~h}$ after the procedure for analgesia. The dogs were anaesthetised with acepromazine $(0.1 \mathrm{mg} / \mathrm{kg}$, s.c.) and propofol (2-8 mg/kg, i.v.) and intubated. After intubation each dog underwent digital disimpaction and tap water enema, was positioned supine, and the abdomen and suprapubic region were shaved. Inhalational anaesthesia (isoflurane 1-2\%) was maintained throughout treatment. Flexible endoscopy was performed with an 8.2-F flexible ureteroscope (Dur-8, Gyrus ACMI) before histotripsy treatment to document a normal lower urinary tract and to serve as an intrasubject control.

TRUS imaging was performed using a Logiq 6 ultrasound scanner (GE Healthcare, Waukesha, WI, USA) with a model ERB probe positioned in a custom holder. Prostatic volume was computed using a stepper volume technique by contouring the prostate margin on transverse slices at $2.5-\mathrm{mm}$ intervals. The therapeutic histotripsy system consists of a 16-element piezoceramic composite array $(750 \mathrm{kHz}, 11 \times$ $14-\mathrm{cm}$ diameter oval shape, focal length $10 \mathrm{~cm}$, focal volume 3 $\times 3 \times 8 \mathrm{~mm}$ (Fig. 1A; Imasonic, Voray sur l'Ognon, France) on a three-axis computer-controlled positioning system (MATLAB, MathWorks, Natick, MA, USA). Coupling was achieved by placing the therapy transducer in a bath of degassed water contained in a plastic membrane in direct contact with the shaved abdomen (Fig. 1B). In all, 21 dogs underwent treatment with histotripsy pulses consisting of five cycle $(6.7 \mu \mathrm{s})$ bursts of acoustic energy delivered at a prescribed PRF for a specified number of pulses. During each treatment, the histotripsy bubble cloud was translated along a single transverse plane that intersected the urethra and periurethral tissues as previously described [17] (Fig. 1C). In
Fig. 1 Histotripsy experimental setup: During treatment the transducer (A) is lowered into a bath of degassed water in direct contact with abdomen (B). Treatment was targeted along a plane transecting the urethra (C).

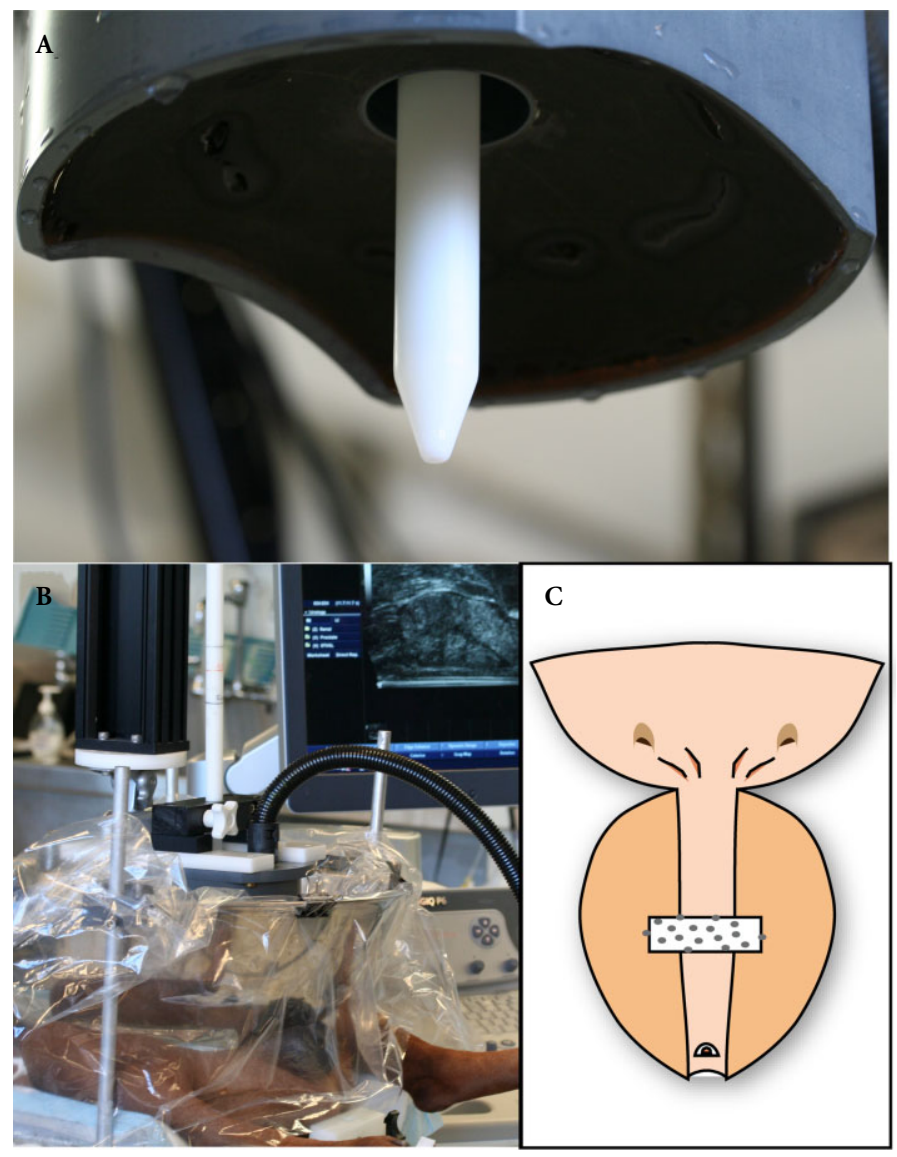

dogs with sufficiently large prostates, two treatments were applied at separate locations at least $1 \mathrm{~cm}$ apart. In the initial phase of the study, treatment consisted of 12 500, 25 000, 50000 , or 100000 pulses/mm of path length at a PRF of $500 \mathrm{~Hz}$ to evaluate the effect of the number of histotripsy pulses on prostatic urethral disintegration. In the second phase of the study, treatment consisted of applying 25000 pulses/mm of path length at PRF of $50,500,2000 \mathrm{~Hz}$ to evaluate the effect of altering PRF on prostatic urethral disintegration. A urinary catheter was not placed after treatment.

After completing histotripsy treatment, cystoscopy was used to evaluate acute changes in urethral appearance. All dogs recovered from anaesthesia and were monitored for treatment-related adverse events. Cystoscopy was repeated on POD 1, 3, 7, and then at euthanasia on POD 14. TRUS was repeated on POD 7 under anaesthesia and at the time of euthanasia with i.v. pentobarbital sodium $140-160 \mathrm{mg} / \mathrm{kg}$. A suprapubic incision was then made and a cystotomy created through which antegrade cystourethroscopy was performed using a 16-F flexible cystoscope (Cy2, Olympus). The prostate, 
bladder and adjacent rectum were surgically removed en bloc and inspected grossly for injury. Tissues were fixed in formalin for 1 week, cut into 5-mm thick slices, dehydrated in $25 \%$ ethanol, paraffin embedded, cut using a microtome in

Fig. 2 TRUS appearance of prostate histotripsy therapy: Early in treatment the histotropsy bubble cloud (arrow) is visualised adjacent to the urethra (outlined circle) (A). During treatment the targeted region becomes progressively hypoechoic producing a hypoechic cavity $\left({ }^{*}\right)$ adjacent to the urethra (B) at the conclusion of treatment.

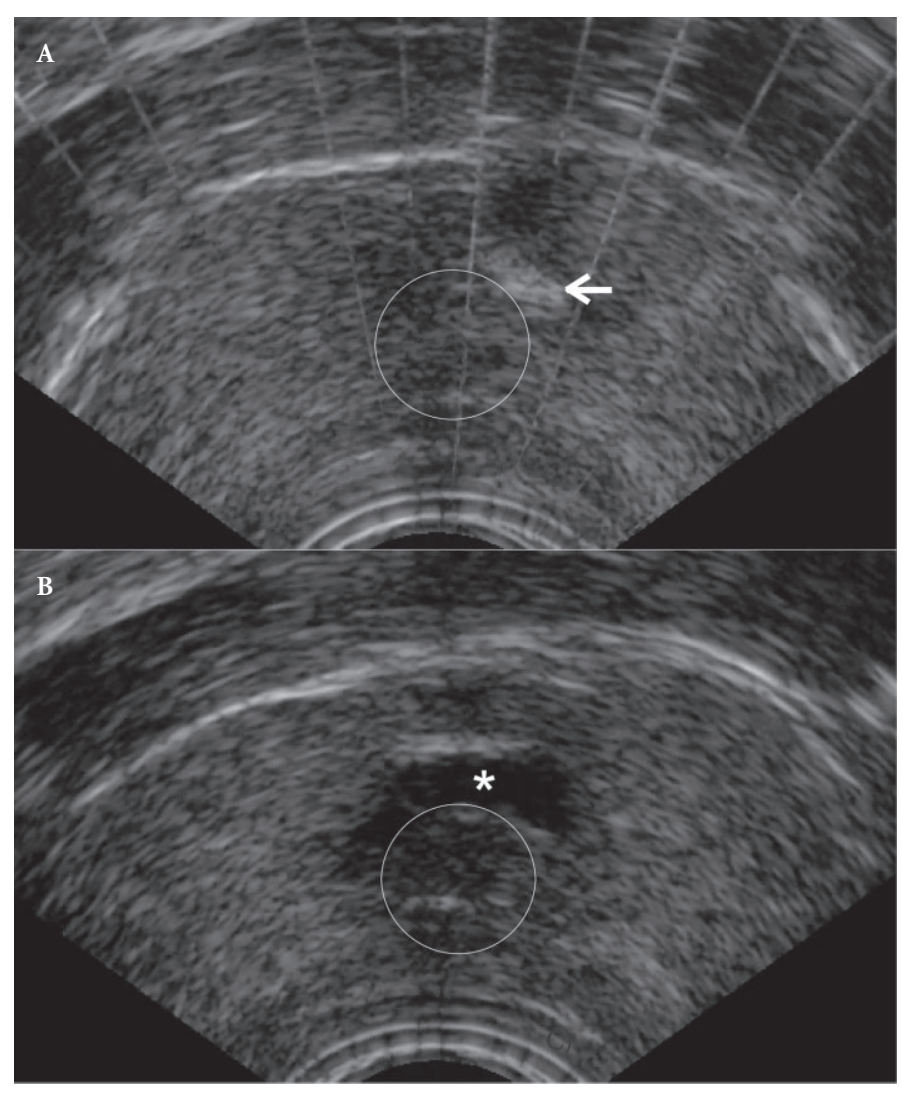

$5-\mu \mathrm{m}$ sections at $1 \mathrm{~mm}$ increments, mounted, and stained with haematoxylin and eosin for histological assessment.

Cumulative rates of urethral disintegration stratified by treatment parameters (number of pulses and PRF) were plotted graphically for interpretation.

\section{Results}

In all, 34 prostate histotripsy treatments were performed in 21 dogs. The mean (range) prostate volume before histotripsy was $22.2(10.5-52.8) \mathrm{mL}$. During treatment, the cavitation bubble cloud was well visualised on TRUS (Fig. 2) and the targeted periurethral parenchymal tissue became progressively hypoechoic, while the prostatic urethra appeared structurally intact throughout treatment despite intraluminal cavitation. Immediately after histotripsy (POD 0), endoscopic assessment of the prostatic urethra/treatment plane showed punctate haemorrhage, shaggy urothelium, tissue flaps and in some cases gross disintegration. In dogs undergoing two treatments, there was an intervening band of normal appearing prostatic urethra between treatments. Acute (POD 0) gross urethral disintegration was endoscopically apparent in only four of 34 (11.8\%) treatment zones immediately after treatment, but developed in 27/34 (79.4\%) by POD 14 .

Treatment planes that did not develop gross urethral disintegration by POD 14 were seen on TRUS to have persistent internal echoes and/or gravity fluid/debris levels within the targeted adjacent periurethral parenchyma suggesting undrained homogenate. Conversely, planes with cavities communicating with disintegrated urethra had absent or minimal internal echoes consistent with drainage of the homogenised material via the urethra. These findings were confirmed histologically (Fig. 3).

Application of more histotripsy pulses (at PRF of $500 \mathrm{~Hz}$ ) resulted in an increased likelihood of urethral disintegration

Fig. 3 Endoscopic appearance of urethral disintegration on POD14 (A) with confirmatory low- (B) and high-power (C) micrographs showing drainage of the homogenised adjacent prostatic parenchyma with minimal inflammation and urothelialisation of the cavity.
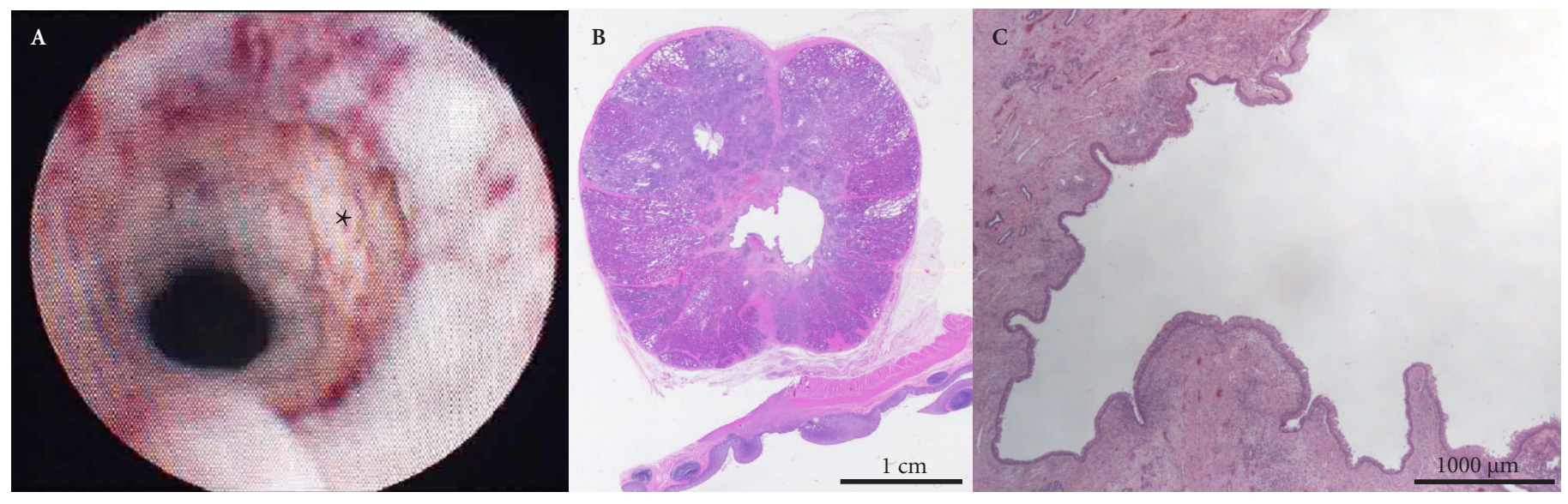
Fig. 4 Cumulative development of prostatic urethral disintegration with histotripsy treatment with 12 500-100 000 pulses/mm with constant PRF $(500 \mathrm{~Hz})$.

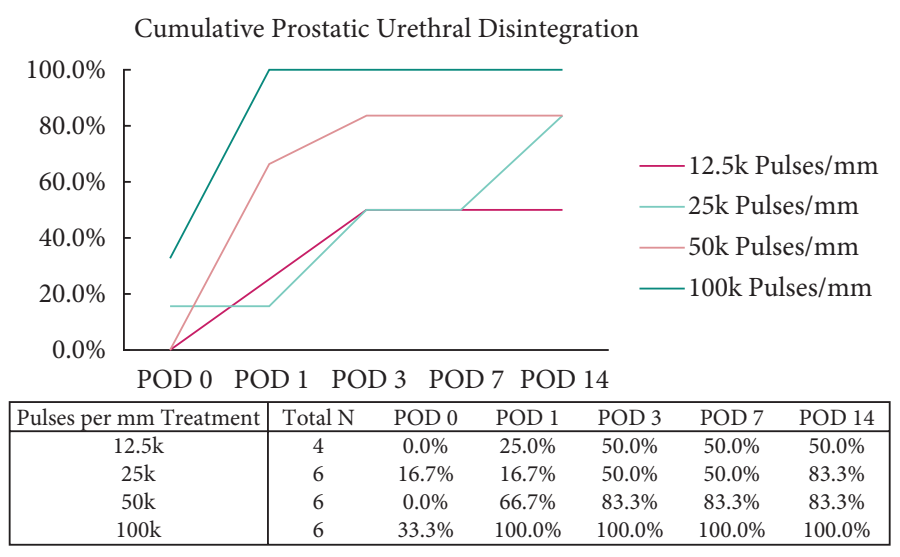

Fig. 5 Cumulative development of prostatic urethral disintegration at PRF of 50,500 , and $2000 \mathrm{~Hz}$ with constant treatment dose (25 000 pulses $/ \mathrm{mm}$ ).

Cumulative Prostatic Urethral Disintegration

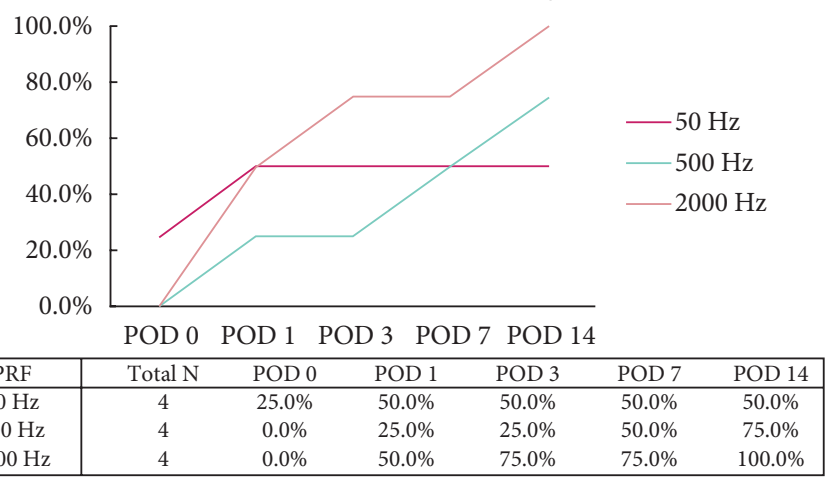

and generally earlier presentation of urethral disintegration (Fig. 4). Specifically, by POD 14 urethral disintegration occurred in $50 \%, 83 \%, 83 \%$, and $100 \%$ of subjects receiving 12 500, 25000,50000 , and 100000 pulses/mm of treatment path, respectively. While immediate prostatic urethral disintegration was rare it did occur within $24 \mathrm{~h}$ of treatment in all the dogs receiving the 100000 pulses/mm treatment. For histotripsy pulse delivery at varying $\operatorname{PRF}(50,500$, and $2000 \mathrm{~Hz}$ ) and constant pulse number (25 000 pulses/mm), increasing treatment PRF to $2000 \mathrm{~Hz}$ was associated with $100 \%$ urethral disintegration by POD 14, compared with $75 \%$ for $500 \mathrm{~Hz}$ and $50 \%$ for $50 \mathrm{~Hz}$ (Fig. 5).

Complications included rectoprostatic fistula in two dogs and development of intraperitoneal urinoma in two dogs secondary to prostatic urethral and capsular perforation.

\section{Discussion}

Over the last several decades, minimally invasive ablative therapies have been introduced in an effort to reduce the morbidity associated with TURP. Previous attempts to accomplish this goal with transurethral needle ablation (TUNA) and microwave forms of energy failed to replicate the efficacy and durability of TURP (possibly due to lack of tissue debulking) $[18,19]$. High-intensity focussed ultrasound (HIFU) has been extensively investigated in Europe and Asia for transrectal treatment of disorders of the prostate. HIFU delivers long (3-5s) pulses of acoustic energy to thermally coagulate the targeted tissue volume [20]. While outcomes are encouraging for prostate cancer [21,22]; previous work attempting to use HIFU for treating $\mathrm{BPH}$ has ultimately proven to be unsuccessful, as nearly $45 \%$ of patients required TURP $\leq 4$ years of HIFU treatment [23]. In this clinical setting, TRUS confirmed TURP-like defects in only $25-30 \%$ of cases after HIFU [24], raising concern about adequate tissue debulking. The authors postulated that three factors may have contributed to the poor durability: (i) inadequate volume of targeted tissue necrosis, (ii) necrotic treatment area evolved into scar possibly increasing fibrosity within the prostate and preventing improvement of urinary symptoms, and (iii) obstructive tissue at the bladder neck was not sufficiently treated to relieve obstruction [23].

In preclinical studies, histotripsy has been shown to homogenise and debulk prostate tissue in the in vivo canine model [11-13]. Based on these results, creating a TURP-like defect with histotripsy appears feasible; however, the optimal treatment strategy to reliably and efficiently achieve this goal is unknown, in part because the prostatic urethra is more resilient to mechanical tissue effects than prostate parenchyma [13]. To best explore prostatic urethral tissue response to a range of acoustic pulse parameters, histotripsy was applied along a linear path perpendicularly traversing the urethra. This simplified the treatment environment (minimising the impact of factors such as acoustic shielding from the pubis and os penis) allowed for multiple treatments per prostate in appropriate subjects. As the size of the cavitation bubble cloud varies with tissue medium, acoustic aperture, and pulse repetition frequency [2] this treatment strategy does not allow calculation of treatment density (pulses/volume treatment) making comparisons of treatment densities in the present study to previous work difficult. Instead, 'histotripsy dose' was reported as the number of pulses applied per mm of transverse treatment path.

Using this treatment strategy with a PRF of $500 \mathrm{~Hz}$, histotripsy doses of 100000 pulses $/ \mathrm{mm}$ treatment were required to achieve urethral disintegration within $24 \mathrm{~h}$ of treatment in all dogs and doses of $\geq 25000$ pulses/mm treatment were required to achieve urethral disintegration in at least $80 \%$ of dogs $\leq 2$ weeks of treatment. Qualitatively, these treatment doses were well above the threshold to induce homogenisation of the adjacent periurethral prostatic parenchyma, which consistently showed increased sensitivity to histotripsy treatment effects compared with the urethra on 
intra-treatment TRUS. Histology confirmed this observation with the presence of undrained homogenised prostatic tissue in all dogs, which failed to achieve disintegration of the adjacent prostatic urethra consistent with previous data $[13,17]$. Further, in such cases, the intact adjacent urethra appeared completely normal despite the adjacent parenchymal damage.

While direct tissue homogenisation is clearly of importance to the mechanism of action of histotripsy [3,6,7], factors other than treatment dose probably contribute to successful tissue homogenisation. One such factor is PRF, as data suggest modifying PRF during histotripsy treatment may alter the efficiency of tissue erosion [25]. Interestingly, in the present study, altering PRF did not appear to substantially impact the development of urethral disintegration $\leq 24 \mathrm{~h}$ of treatment. However, there was an increase in urethral disintegration 14 days after treatment as PRF was increased from 50 to 500 to $2000 \mathrm{~Hz}$ ( $50 \%$ vs $75 \%$ vs $100 \%$, respectively). This observation, if confirmed by larger studies, was unexpected based on in vitro data suggesting that increased $\mathrm{PRF}$ is associated with decreased efficiency of tissue homogenisation, sporadic cavitary damage, and increased number of pulses to achieve equivalent damage in both myocardium and liver [16,26]. One possible explanation for the greater rate of delayed urethral disintegration at higher PRF is that the larger bubble-cloud produced by increasing PRF may lead to a larger field of incompletely homogenised, yet lethal tissue damage, that when remodelled in the chronic setting leads to overall higher rates of urethral disintegration.

While the prostatic urethra is more resistant to histotripsy than the parenchyma/adenoma, the basis of its resistance is unknown. In vitro studies, examining lesion size in agar have shown that lesion size is directly related to duration of treatment (number of pulses) and inversely related to 'tissue' stiffness [15]. Histologically, the prostatic urethra (and its periurethral fibromuscular) stroma contains more collagen than the surrounding prostatic parenchyma, suggesting increased tissue strength and 'stiffness' compared with the parenchyma. As a result, the increased collagen content of the prostatic urethra may contribute to the higher threshold for histotripsy induced tissue disintegration, a hypothesis that is actively being evaluated. Aside from decreased sensitivity of the urethra to the effects of histotripsy, an additional possible explanation of failure to disintegrate the urethra is inadequate urethral targeting. However, using real-time

cystourethroscopy, we have previously shown the presence of histotripsy induced intraluminal cavitation using the same treatment strategy [17] suggesting adequate urethral targeting.

Although the results from the present study will help guide future histotripsy research, they are not without limitations, most importantly their generalizability to humans. However, the prostate is very similar between both species with the arterial supply originating from the internal pudendal artery, and branches perforating the capsule to supply the stroma and glandular tissues. Structurally, while the canine prostate is bi-lobed without zonal structures, the human prostate is subdivided into several zones, with potentially different treatment thresholds, with the transition zone being most relevant to $\mathrm{BPH}$ [27]. Additionally, the intraperitoneal location of the canine prostate lends itself to a transabdominal histotripsy approach, whereas structural modelling suggests that the human prostate will probably be more amenable to a transperineal approach [28]. The transabdominal approach combined with small prostate size probably contributed to the complications in the present study - the cigar shaped histotripsy bubble cloud was perpendicular to the axis of the urethra making adequate targeting of the urethra while attempting to maintain the bubble cloud within the parenchyma along the midline sulcus difficult in some dogs, thus increasing the risk of rectal injury and prostatic capsular perforation. Ultimately, human studies are needed to determine both the clinical efficacy and optimal treatment algorithm for prostate histotripsy for $\mathrm{BPH}$.

In summary, extracorporeal application of histotripsy to the prostate is capable of homogenising targeted prostate parenchyma with subsequent creation of a TURP-like defect. Increasing the number of histotripsy pulses and/or increasing the PRF of these pulses applied to the urethra appears to improve the rate and efficiency of prostatic urethral disintegration in the canine model, which may facilitate drainage of the homogenate and enhance prostate debulking. This understanding will prove useful in developing treatment strategies for prostate histotripsy treatment of $\mathrm{BPH}$ in human trials.

\section{Disclosures}

W.W.R. and T.L.H. have equity, royalty, and consulting interests in HistoSonics, Inc.

Funding: NIH RO1DK087871.

\section{Conflict of Interest}

T.L.H. and W.W.R. report grants from National Institutes of Health, during the conduct of the study; personal fees and other from HistoSonics, Inc., outside the submitted work. In addition, T.L.H. and W.W.R have a patent US 8,057,408,B2 licensed to HistoSonics, and a patent PCT/2115-003767/POA pending to HistoSonics.

G.R.S., N.R.S. and K.A.I. have nothing to disclose.

\section{References}

1 Kieran K, Hall TL, Parsons JE et al. Refining histotripsy: defining the parameter space for the creation of nonthermal lesions with high intensity, pulsed focused ultrasound of the in vitro kidney. J Urol 2007; 178: $672-6$ 
2 Xu Z, Hall TL, Fowlkes JB, Cain CA. Effects of acoustic parameters on bubble cloud dynamics in ultrasound tissue erosion (histotripsy). J Acoust Soc Am 2007; 122: 229-36

3 Xu Z, Raghavan M, Hall TL et al. High speed imaging of bubble clouds generated in pulsed ultrasound cavitational therapy - histotripsy. IEEE Trans Ultrason Ferroelectr Freq Control 2007; 54: 2091-101

$4 \mathrm{Xu}$ Z, Hall TL, Fowlkes JB, Cain CA. Optical and acoustic monitoring of bubble cloud dynamics at a tissue-fluid interface in ultrasound tissue erosion. J Acoust Soc Am 2007; 121: 2421-30

5 Xu Z, Raghavan M, Hall TL, Mycek MA, Fowlkes JB. Evolution of bubble clouds induced by pulsed cavitational ultrasound therapy histotripsy. IEEE Trans Ultrason Ferroelectr Freq Control 2008; 55: 1122-32

6 Tran BC, Seo J, Hall TL, Fowlkes JB, Cain CA. Microbubble-enhanced cavitation for noninvasive ultrasound surgery. IEEE Trans Ultrason Ferroelectr Freq Control 2003; 50: 1296-304

7 Xu Z, Fowlkes JB, Rothman ED, Levin AM, Cain CA. Controlled ultrasound tissue erosion: the role of dynamic interaction between insonation and microbubble activity. J Acoust Soc Am 2005; 117: 424-35

8 Xu Z, Fan Z, Hall TL, Winterroth F, Fowlkes JB, Cain CA. Size measurement of tissue debris particles generated from pulsed ultrasound cavitational therapy-histotripsy. Ultrasound Med Biol 2009; 35: 245-55

9 Hall TL, Fowlkes JB, Cain CA. A real-time measure of cavitation induced tissue disruption by ultrasound imaging backscatter reduction. IEEE Trans Ultrason Ferroelectr Freq Control 2007; 54: 569-75

10 Wang TY, Xu Z, Winterroth F et al. Quantitative ultrasound backscatter for pulsed cavitational ultrasound therapy- histotripsy. IEEE Trans Ultrason Ferroelectr Freq Control 2009; 56: 995-1005

11 Lake AM, Hall TL, Kieran K, Fowlkes JB, Cain CA, Roberts WW. Histotripsy: minimally invasive technology for prostatic tissue ablation in an in vivo canine model. Urology 2008; 72: 682-6

12 Hempel CR, Hall TL, Cain CA, Fowlkes JB, Xu Z, Roberts WW. Histotripsy fractionation of prostate tissue: local effects and systemic response in a canine model. J Urol 2011; 185: 1484-9

13 Hall TL, Hempel CR, Wojno K, Xu Z, Cain CA, Roberts WW. Histotripsy of the prostate: dose effects in a chronic canine model. Urology 2009; 74: 932-7

14 Wheat JC, Hall TL, Hempel CR, Cain CA, Xu Z, Roberts WW. Prostate histotripsy in an anticoagulated model. Urology 2010; 75: 207-11

$15 \mathrm{Xu}$ J, Bigelow TA. Experimental investigation of the effect of stiffness, exposure time and scan direction on the dimension of ultrasound histotripsy lesions. Ultrasound Med Biol 2011; 37: 1865-73

16 Parsons JE, Cain CA, Abrams GD, Fowlkes JB. Pulsed cavitational ultrasound therapy for controlled tissue homogenization. Ultrasound Med Biol 2006; 32: 115-29
17 Schade GR, Styn NR, Hall TL, Roberts WW. Endoscopic assessment and prediction of prostate urethral disintegration after histotripsy treatment in a canine model. J Endourol 2012; 26: 183-9

18 McVary KT, Roehrborn CG, Avins AL et al. Guidline on the Managment of Benign Prostatic Hyperplasia (BPH). American Urologic Association; 2010 [cited 5 April 2011]. Available at: http://www.auanet.org/education/ guidelines/benign-prostatic-hyperplasia.cfm. Accessed July 2013

19 McVary KT, Roehrborn CG, Avins AL et al. Update on AUA guideline on the management of benign prostatic hyperplasia. J Urol 2011; 185: 1793-803

20 Hynynen K, Darkazanli A, Damianou CA, Unger E, Schenck JF. Tissue thermometry during ultrasound exposure. Eur Urol 1993; 23 (Suppl. 1): 12-6

21 Ahmed HU, Freeman A, Kirkham A et al. Focal therapy for localized prostate cancer: a phase I/II trial. J Urol 2011; 185: 1246-54

22 Crouzet S, Rebillard X, Chevallier D et al. Multicentric oncologic outcomes of high-intensity focused ultrasound for localized prostate cancer in 803 patients. Eur Urol 2010; 58: 559-66

23 Madersbacher S, Schatzl G, Djavan B, Stulnig T, Marberger M. Long-term outcome of transrectal high- intensity focused ultrasound therapy for benign prostatic hyperplasia. Eur Urol 2000; 37: 687-94

24 Madersbacher S, Kratzik C, Susani M, Marberger M. Tissue ablation in benign prostatic hyperplasia with high intensity focused ultrasound. J Urol 1994; 152: 1956-61

25 Xu Z, Ludomirsky A, Eun LY et al. Controlled ultrasound tissue erosion. IEEE Trans Ultrason Ferroelectr Freq Control 2004; 51: 726-36

26 Wang TY, Xu Z, Hall TL, Fowlkes JB, Cain CA. An efficient treatment strategy for histotripsy by removing cavitation memory. Ultrasound Med Biol 2012; 38: 753-66

27 Evans HE. Miller's Anatomy of the Dog. Philadelphia, PA: W. B. Saunders, 1993: 514-6

28 Hall TL, Hempel CR, Sabb BJ, Roberts WW. Acoustic access to the prostate for extracorporeal ultrasound ablation. J Endourol 2010; 24: 1875-81

Correspondence: William W. Roberts, University of Michigan, Department of Urology, TC 3879, 1500 E. Medical Center Dr, Ann Arbor, MI 48109, USA.

e-mail: willrobe@med.umich.edu

Abbreviations: HIFU, high-intensity focussed ultrasound; POD, postoperative day; PRF, pulse-repetition frequency. 